\title{
Screening for diabetic retinopathy: new perspectives and challenges
}

Stela Vujosevic, Stephen J Aldington, Paolo Silva, Cristina Hernández, Peter Scanlon, Tunde Peto, Rafael Simó

\begin{abstract}
Although the prevalence of all stages of diabetic retinopathy has been declining since 1980 in populations with improved diabetes control, the crude prevalence of visual impairment and blindness caused by diabetic retinopathy worldwide increased between 1990 and 2015, largely because of the increasing prevalence of type 2 diabetes particularly in low-income and middle-income countries. Screening for diabetic retinopathy is essential to detect referable cases that need timely full ophthalmic examination and treatment to avoid permanent visual loss. In the past few years, personalised screening intervals that take into account several risk factors have been proposed, with good cost-effectiveness ratios. However, resources for nationwide screening programmes are scarce in many countries. New technologies, such as scanning confocal ophthalmology with ultrawide field imaging and handheld mobile devices, teleophthalmology for remote grading, and artificial intelligence for automated detection and classification of diabetic retinopathy, are changing screening strategies and improving cost-effectiveness. Additionally, emerging evidence suggests that retinal imaging could be useful for identifying individuals at risk of cardiovascular disease or cognitive impairment, which could expand the role of diabetic retinopathy screening beyond the prevention of sight-threatening disease.
\end{abstract}

\section{Introduction}

Diabetic retinopathy remains the leading cause of vision loss and preventable blindness in adults aged 20-74 years, particularly in middle-income and high-income countries. In a meta-analysis of 35 studies done worldwide between 1980 and 2008, researchers estimated an overall prevalence of $34.6 \%(95 \%$ CI $34.5-34 \cdot 8)$ for any diabetic retinopathy, $6 \cdot 96 \%(6 \cdot 87-7 \cdot 04)$ for proliferative diabetic retinopathy, $6.81 \%(6.74-6.89)$ for diabetic macular oedema, and $10 \cdot 2 \%(10 \cdot 1-10 \cdot 3)$ for vision-threatening diabetic retinopathy among people with diabetes. ${ }^{2}$ Prevalence of any diabetic retinopathy and proliferative diabetic retinopathy was higher in people with type 1 diabetes than in people with type 2 diabetes. $^{2}$

Although the proportion of people with diabetes developing proliferative diabetic retinopathy and severe visual loss has been declining between 1980 and 2008 in populations with improved diabetes control, ${ }^{3}$ the crude prevalence of visual impairment and blindness caused by diabetic retinopathy increased substantially between 1990 and 2015 according to the latest report of the Vision Loss Expert Group of the Global Burden of Disease Study, ${ }^{4}$ largely because of the increasing prevalence of type 2 diabetes in low-income and middle-income countries. Thus, the number of people affected by blindness due to diabetic retinopathy increased from $0 \cdot 2$ million to 0.4 million, and moderate to severe vision impairment increased from 1.4 million to 2.6 million. ${ }^{4}$ Furthermore, it has been estimated that the number of people with diabetes affected by any diabetic eye disease in Europe will increase from 6.4 million in 2019 to 8.6 million in 2050 , and that $30 \%$ of affected individuals will require close monitoring or treatment. ${ }^{5}$

Few population-based studies examining the incidence of diabetic retinopathy have been done since 2000. The incidence of diabetic retinopathy was higher in studies from before 2000 than in those reported after $2000{ }^{6}$
However, contemporary studies that include more data from low-income and middle-income countries are needed.

Screening for diabetic retinopathy is necessary to detect referable cases that need timely full ophthalmic examination and treatment to avoid permanent visual loss. However, the resources for nationwide screening programmes are not sufficient in many countries. The new technologies based on artificial intelligence, which permit to implement personalised predictive models, the use of telemedicine, and portable imaging devices, are changing the screening strategies and are improving the cost-effectiveness of screening. In this Review, these new tools-which are changing the landscape of screening strategies-will be analysed. In addition, we will comment on the possibility of using retinal examination to identify patients at risk of cardiovascular disease and cognitive impairment, thus expanding the role of the screening of diabetic retinopathy.

\section{Risk factors for diabetic retinopathy}

The most relevant risk factors for the development of diabetic retinopathy are the duration of diabetes, poor glycaemic control (high $\mathrm{HbA}_{1 \mathrm{c}}$ and the presence of hypertension. Notably, blood glucose control has a stronger effect than blood pressure control on the risk of developing diabetic retinopathy. ${ }^{7-10}$

Other risk factors for diabetic retinopathy include dyslipidaemia, high BMI, puberty, pregnancy, and cataract surgery. ${ }^{2}$ However, clinical studies on patients living with diabetes have shown substantial variation in the onset and by known risk factors, ${ }^{7}$ and clinicians are aware that not all patients with poor control of glycemia or uncontrolled blood pressure develop diabetic retinopathy. Similarly, some patients with good glycaemic control and without hypertension can develop diabetic retinopathy. An analysis severity of diabetic retinopathy that is not fully explained
Lancet Diabetes Endocrinol 2020 Published Online February 27, 2020 https://doi.org/10.1016/ S2213-8587(19)30411-5

Eye Unit, University Hospital Maggiore della Carità, Novara, Italy (S Vujosevic MD); Department of Ophthalmology, Gloucestershire Hospitals NHS Foundation Trust, Cheltenham, UK (S) Aldington HND PScanlon MD); Beetham Eye Institute, Joslin Diabetes Centre, Harvard Medical School, Boston, MA, USA (P Silva MD); Philippine Eye Research Institute, University of the Philippines, Manila, Philippines (P Silva MD); Diabetes and Metabolism Research Unit, Vall d'Hebron Research Institute, Barcelona, Spain (C Hernández MD, Prof R Simó MD); Department of Medicine and Endocrinology, Autonomous University of Barcelona, Barcelona, Spain (C Hernández, Prof R Simó); Centro de Investigación Biomédica en Red de Diabetesy Enfermedades Metabólicas Asociadas, Instituto de Salud Carlos III, Madrid, Spain (C Hernández MD, Prof R Simó MD); and Centre for Public Health, Queen's University Belfast, Belfast, UK (T Peto MD)

Correspondence to: Prof Rafael Simó, Diabetes and Metabolism Research Unit, Vall d'Hebron Research Institute, 08035 Barcelona, Spain rafael.simo@vhir.org 
Panel: Diabetes screening recommendations

ADA recommendations for timing of eye examination for different types of diabetes

- Type 1 diabetes: within 5 years after onset of diabetes

- Type 2 diabetes: at time of diagnosis

- Women with pre-existing diabetes who plan pregnancy or who have become pregnant: before pregnancy or in the first trimester, and then monitored every trimester and for 1 year post partum, as indicated by the degree of retinopathy

- Gestational diabetes: not required

Recommendations for eye examination components

- The $\mathrm{ICO}^{33}$ recommends two minimum screening examination components to ensure appropriate referral:

- A screening vision examination, which can be done in any of the following ways, depending on resource availability:

- Refracted visual acuity examination with $3 \mathrm{~m}$ or $4 \mathrm{~m}$ visual acuity lane and a high-contrast visual acuity chart

- Visual acuity examination with a near or distance eye chart and a pinhole option if visual acuity is reduced

- Visual acuity examination with $6 / 12$ (20/40) equivalent handheld chart consisting of at least five standard letters or symbols and a pinhole option if visual acuity is reduced

- A retinal examination, which can be done in either of the following ways:

- Direct or indirect ophthalmoscopy or slit-lamp biomicroscopy of the retina

- Retinal (fundus) photography with any of the following: 30-degree to wide field, mono or stereo photography, and dilated or undilated photography; can be done remotely (telemedicine approaches)

- The $\mathrm{ADA}^{34}$ recommends that patients should have an initial dilated and comprehensive eye examination by an ophthalmologist or optometrist

$\mathrm{ADA}=$ American Diabetes Association. $\mathrm{ICO}=$ International Council of Ophthalmology.

of the Diabetes Control and Complications Trial and the Epidemiology of Diabetes Interventions and Complications study showed that $\mathrm{HbA}_{1 c}$ values accounted for up to $11 \%$ of the risk of developing diabetic retinopathy, with the unexplained $89 \%$ of variation in risk is due to elements of the diabetic milieu not captured by the mean $\mathrm{HbA}_{1 \mathrm{c}}$ value.? These data suggest that other factors such as glucose variability ${ }^{11,12}$ and genetic determinant ${ }^{13-16}$ have a relevant role in the development and progression of diabetic retinopathy.

\section{The cost-effectiveness of screening}

Several studies from different countries around the world have been done to investigate the cost-effectiveness of screening for diabetic retinopathy, especially vision-threatening retinopathy. ${ }^{17-19}$ Cost-effectiveness of population-based screening programmes is heavily dependent on the frequency of retinal examinations and retinal imaging. ${ }^{20}$ Extending the screening interval from annual to every 2 or 3 years in patients with diabetes who had no evidence of any retinopathy at first eye examination has been reported to be cost-effective in several European studies. ${ }^{21-24}$ Importantly, differentiating patients into low-risk and high-risk groups has the potential to further improve cost-effectiveness. . $4,26,27$

Screening programmes for diabetic retinopathy with 2D non-stereoscopic digital fundus photography are currently implemented in primary care. However, interpretation of the photographs requires specialised knowledge and expertise in diabetic eye disease. Since the number of patients with diabetes is rising rapidly, manual grading for diabetic retinopathy in screening programmes will not be sustainable. In this context, the emerging use of validated automated grading software will undoubtedly have a beneficial effect on the cost-effectiveness of screening. ${ }^{28,29}$ More controversial is the use of ultrawide field imaging systems for the assessment of additional peripheral retinal lesions with major risk of diabetic retinopathy progression. ${ }^{30,31}$ Regarding the screening of diabetic macular oedema, the use of optical coherence tomography for first-line screening has yet to be fully justified from the financial perspective. ${ }^{17}$ However, optical coherence tomography has been shown to be costeffective for second-line screening in patients with positive 2D markers at primary screening. ${ }^{32}$

\section{Screening for diabetic retinopathy: who, when, and how Current guidelines and procedures}

The most recent guidelines and procedures for diabetic retinopathy screening were reported by the International Council of Ophthalmology in 2018 as part of their guidelines for diabetic eye care, ${ }^{33}$ and by the American Diabetes Association in the same year as part of their position statement on diabetic retinopathy. ${ }^{34}$ The American Diabetes Association recommends a well-defined first eye examination with different timing depending on the type of diabetes, supported by a moderate level (level B) of evidence (panel). To ensure appropriate referral to an ophthalmologist, the minimum screening examination would need to include vision screening and retinal examination adequate for classification of diabetic retinopathy. ${ }^{34}$ The International Council of Ophthalmology classification for diabetic retinopathy and diabetic macular oedema considers five stages of retinopathy (none, mild non-proliferative, moderate non-proliferative, severe nonproliferative, and proliferative; figure 1A). The classification for diabetic macular oedema has been updated, with the classifications consisting of no diabetic macular oedema, non-centre-involving diabetic macular oedema, and centreinvolving diabetic macular oedema (figure 1B). This 
classification is the most widely used in the clinical practice. $^{33}$

Retinal examination includes either retinal photography or fundus examination (ophthalmoscopy). Mydriatic and non-mydriatic colour retinal photography have shown better sensitivity in diabetic retinopathy screening than direct and indirect ophthalmoscopy. ${ }^{35,36}$ Multifield (three fields) non-mydriatic imaging has been shown to have a good agreement with dilated ophthalmoscopy or seven Early Treatment Diabetic Retinopathy Study (ETDRS) fields, ${ }^{37}$ but not when only one-field or two-field non-mydriatic 45-degree images are used. ${ }^{37,38}$ Currently, there is no agreement regarding the number of non-mydriatic fundus fields required to reliably detect and grade diabetic retinopathy and diabetic macular oedema in telemedicine screening programmes. In the past, seven standard mydriatic and stereoscopic 30-degree fundus fields (seven-field ETDRS) were considered the gold standard for fundus assessment in clinical trials in diabetic retinopathy. However this procedure is time-consuming and expensive, and thus not appropriate in the screening setting. Therefore, fewer fundus fields (three, two, or even one central field, varying from 45 to 60 degrees) have been proposed for diabetic retinopathy and diabetic macular oedema screening. ${ }^{39-42}$ Data suggest a sensitivity of $80-98 \%$ and specificity of $86-100 \%$ for a limited number of fundus fields (two to four) versus seven ETDRS fields for detecting any diabetic retinopathy. By contrast, lower sensitivity (54-78\%) and specificity $(88-89 \%)$ was recorded when use of single central field come compared with seven ETDRS fields. ${ }^{40,43}$ The major clinical value of diabetic retinopathy screening is to detect referable cases that need timely full ophthalmic examination and treatment to avoid permanent visual loss. For this reason, high sensitivity values $(>80 \%)$ are required for an effective screening programme; as such, one non-mydriatic 45-degree central-field photography is not suitable for a community-based screening programme. ${ }^{34}$ Ultrawide field systems covering up to 200 degrees of the fundus have also been used for diabetic retinopathy screening (figure 2). With ultrawide field imaging systems, pharmacological mydriasis might be required for single retinal lesion assessment to decrease the proportion of ungradable images (from $4.5 \%$ to $0 \%$ ). ${ }^{45}$

To be useful for patients, a screening programme should be coordinated with access to adequate and timely referral to the ophthalmologist. The timing of next screening visits and referral to the ophthalmologist in both high-resource and medium-resource or lowresource settings are shown in the table.

\section{Development of nationwide screening programmes}

The principles of screening for human diseases, which were derived from the public health papers produced by the WHO in 1968, include the proposition that "the cost

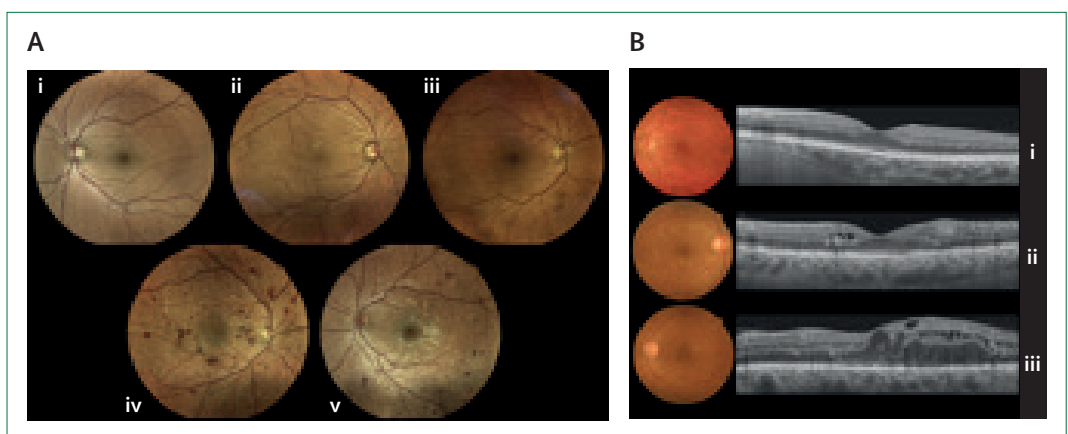

Figure 1: Retinal images corresponding to the different stages of diabetic retinopathy and diabetic macular oedema

(A) Colour fundus images (covering about 60 central degrees, obtained with true colour confocal scanning ophthalmoscope) representative of the five stages of diabetic retinopathy classification according to the International Council of Ophthalmology classification: (i) no diabetic retinopathy; (ii) mild non-proliferative diabetic retinopathy; (iii) moderate non-proliferative diabetic retinopathy; (iv) severe non-proliferative diabetic retinopathy; and ( $v$ ) proliferative diabetic retinopathy with new vessels on the disc. (B) Colour fundus images (left) and optical coherence tomography images (right) representative of the three stages of diabetic macular oedema classification according to the International Council of Ophthalmology classification: (i) no diabetic macular oedema; (ii) non-centre-involving diabetic macular oedema; and (iii) centre-involving diabetic macular oedema.

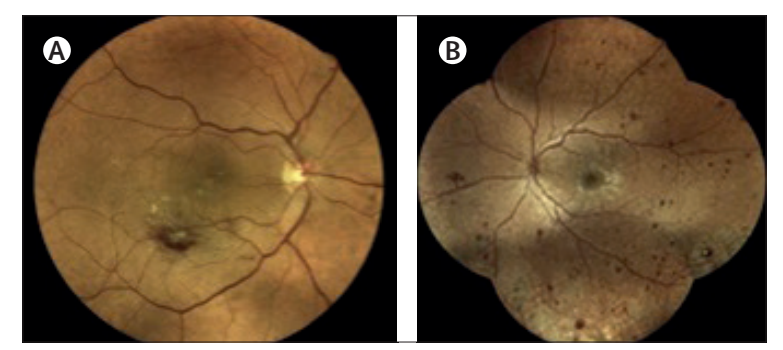

Figure 2: Comparison between simple central fundus photography and ultrawide field composite image

(A) Central 60-degree fundus field of the right eye showing centre-involving diabetic macular oedema with moderate non-proliferative diabetic retinopathy. (B) Ultrawide field composite image covering 150 degrees of the left eye, showing proliferative diabetic retinopathy (with new vessels on the disc, numerous haemorrhages, and venous beading) with some peripheral lesions due to laser treatment.

of the case-finding programme (including early diagnosis and treatment of patients diagnosed) should be economically balanced in relation to possible expenditure on medical care as a whole". ${ }^{46}$ Hence, even in resourcerich countries, the frequency of invited screening and uptake by those individuals who most need to attend is compromised by a relative or absolute lack of resources and by a reluctance in some patients to recognise the importance of screening. ${ }^{47-52}$ Equally, regular annual screening of patients with a very low risk of developing any serious complications is of questionable value, both economically and socially. ${ }^{48,49}$ However, such repeated screening does provide reassurance that the condition is not progressing for the committed, well-controlled but worried patients. ${ }^{53}$

Diabetic eye screening, frequently done at a time when a patient attends other investigations (ie, opportunistic screening), has been successfully implemented in many locations and regions; many of these opportunistic screening practices have become well established and 


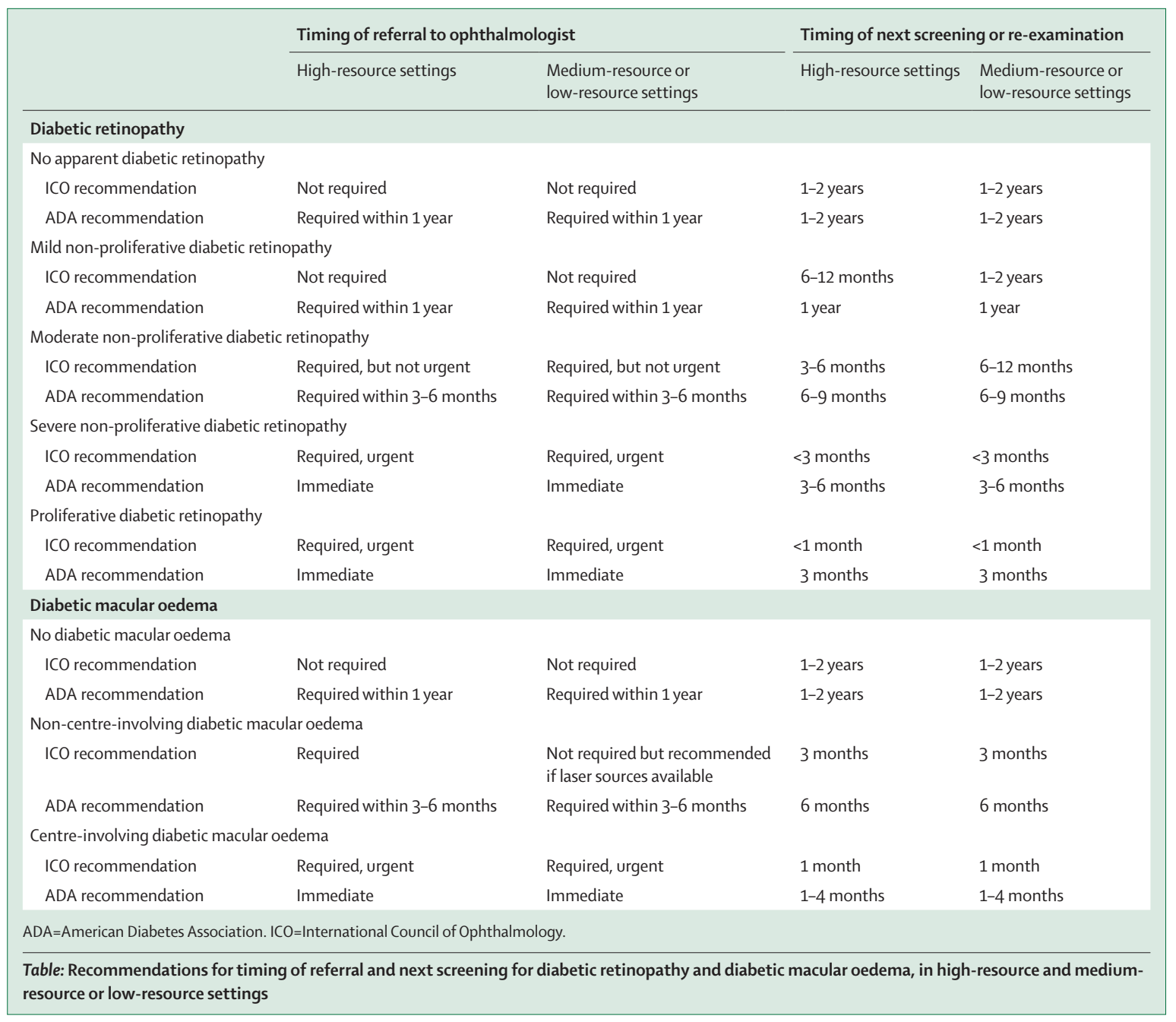

offer high quality. The caveat, however, is that these screenings are most often only done in a specifically defined local population. By contrast, systematic screening, which aims to include the whole population at risk in its target group, is far more complex to implement and sustain, yet has been successfully realised in some locations.

True nationwide population-based systematic diabetic eye screening programmes only exist in Iceland, , $^{5,55}$ the $U K,{ }^{47,56}$ and Ireland. ${ }^{57}$ However, many parts of mainland Europe (particularly Norway, Denmark, Sweden, the Netherlands, Czech Republic, Italy, Poland, Serbia, Hungary, and Turkey) have made substantial strides towards establishing and providing regional or even superregional screening and treatment services..$^{20,58-60}$ Parts of Africa (particularly Botswana) and Asia (particularly China, Singapore, Indonesia, and Bangladesh) are also making good progress, although much still remains to be done.

Several factors are necessary for the successful implementation of systematic screening programmes, including equitable and widespread access to laser treatment and trained staff (including administrative staff), together with local screening programmes that can expand to regions and then to entire nations. Countries with the lowest financial resources, decentralised health care, or mainly private insurance-based health care, encounter barriers to implementation.

When systematic screening for diabetic retinopathy was introduced in the four UK nations, all eligible patients were theoretically offered screening on an annual basis, until such time as they required referral to a hospital eye specialist, or they became functionally blind in both eyes or too unwell to attend. ${ }^{47}$ Since then, several issues have become clear. First, an ever-increasing eligible patient population ( $5 \%$ increase per year) in the environment of reduced budgets is not sustainable. ${ }^{16,18,27,61-64}$ Second, a large proportion of screened patients has the same result showing no detected retinopathy every year, and are demonstrably at very low risk of future vision loss..$^{27,4,45}$ Third, there is a frustrating ceiling of less than $85 \%$ uptake of annual eye screening invitations in the English National 
Health Service Diabetic Eye Screening Programme (key performance indicator data 2018-19). ${ }^{65}$ Fourth, nonattendance at screening substantially increases the risk of the subsequent detection of vision-threatening retinal features..$^{52,66}$ Finally, the greatest effects can be achieved when screening is offered at locations and times that match the needs of the patient, not the provider. ${ }^{67-69}$ These findings might be particularly relevant in resource-poor settings and in locations where systematic screening is being introduced..$^{35}$

A key message is that organisers of programmes that are being designed or initiated should learn from the mistakes made in the creation of established screening programmes. Fixed annual screening for all people with diabetes, irrespective of risk of future visual loss, is demonstrably not deliverable or sustainable; centralised registers of eligible people are essential and need constant updates; buy-in from health-care professionals, patients, and their families is crucial; accurate data collection, review, and reporting-including managing patients who do not attend or are lost to follow-up or treatment-is essential to reduce vision loss and blindness in people at the highest risk.

Retinal screening, if done only by a specialist ophthalmologist's clinical examination, can rarely be shown to be cost-effective or to produce an auditable result. In some circumstances, however, opportunistic diabetic retinopathy screening via direct patient examinationsupported by immediate access to treatment, education, and support-is the only viable option in rural and remote communities.

In nationwide screening programmes, skilled staff, access to treatment, financial resources, and appropriate health-care professional and patient education should be in place to reduce the global burden of diabetic retinopathy. Additionally, novel strategies for engaging and integrating primary and secondary health-care providers appropriately to ensure timely diagnosis, referral, and treatment of diabetic retinopathy is essential to improve populationlevel coverage and cost-effectiveness. ${ }^{70,71}$

However, provision of the screening assessment is only a small part of the full programme for reducing the risk of visual loss associated with development of diabetic retinopathy. Eye screening as an isolated event, if separated from diabetes management, ${ }^{68}$ specialist ophthalmic services, ${ }^{47}$ and patient understanding and self-management of their condition (with relevant caveats and limitations), is almost certainly bound to fail. .8,49,72 $^{4}$

\section{The changing landscape of screening strategies Novel methods of retinal imaging for ocular telehealth programmes}

Recent technological advances in diabetic retinopathy screening fall into three categories: image capture, image analysis, and risk assessment. Novel methods of image capture include the use of scanning (laser) confocal ophthalmoscope-based cameras with ultrawide field imaging or conventional cameras with improvements, such as the use of handheld mobile devices. Automated image analysis and use of artificial intelligence can make an important contribution in teleophthalmology not only for the automated detection of diabetic retinopathy but also to identify patients at risk of cardiovascular or neurodegenerative diseases (see section "Retinopathy screening and other diabetes complications"). New methods for risk assessment include the use of alternative methods for screening (eg, tear film and potentially mRNA and genetic information) and visual function data. However, before implementation of these new technologies, evidence is needed for their clinical effectiveness and costeffectiveness.

The use of scanning confocal ophthalmoscopy (with lasers or a white-light light-emitting diode illuminator), particularly with ultrawide field imaging technology, can potentially improve image quality and the field of view without the need for pupillary dilation. ${ }^{73}$ In a nationwide teleophthalmology programme for diabetic retinopathy in the setting of the Indian Health Service-Joslin Vision Network programme (which serves American Indian and Alaska Native communities at 97 sites across 25 states), it was shown that non-mydriatic ultrawide field imaging was able to considerably reduce the rate of ungradable images and substantially increase not only the detection of diabetic retinopathy, but also of referable diabetic retinopathy, compared with non-mydriatic multifield fundus imaging. ${ }^{74}$ Additionally, the presence of predominantly peripheral lesions seen only with ultrawide field images was shown in one study to enable the identification of a more severe level of diabetic retinopathy in $7.2 \%$ of eyes $(9.6 \%$ of patients), suggesting an increased risk of retinopathy progression in these eyes. ${ }^{30}$ These findings support the substantial potential advantages of ultrawide field imaging for large diabetic retinopathy screening teleophthalmology programmes.

\section{Approaches to retinal image analysis and prediction of retinopathy progression}

The use of artificial intelligence models for retinal image interpretation in the screening of diabetic retinopathy is a rapidly evolving field. ${ }^{75,76}$ Historically, artificial intelligence systems have relied on so-called hard-coded image processing and specific lesion-detection algorithms. In the past decade, computing advances have led to impressive results with deep learning processes that enable artificial intelligence systems to self-learn and improve with the increasing number of images assessed. Leveraging current image databases tens of thousands to hundreds of thousands of images, deep learning algorithms have now surpassed traditional machine-learning methods. The use of deep learning has led to substantial improvements in diabetic retinopathy detection, achieving significantly higher sensitivity (87-90\%) and specificity $(98 \%) .7$

In traditional machine learning, features needed to be extracted manually with specific feature-detection 


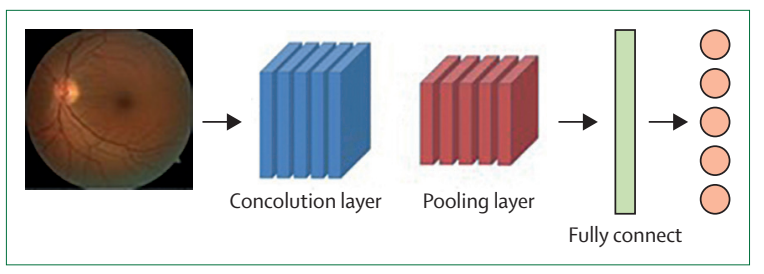

Figure 3: Structural design of a basic convolutional neural network The basic structural design of a convolutional neural network includes convolution, pooling, and a fully connected layer. A convolutional neural network is a deep-learning algorithm that can take in an input image, assign importance to different features in an image, and is able to differentiate one feature from another. This self-learning approach removes the need to explicitly identify the features in images, which made retinal image analysis difficult to automate in the past. The typical convolutional neural network contains paralle mechanisms of convolution and pooling operations alternating to one another. The output from the convolution and pooling layers represents high-level features of the input image. The purpose of the fully connected layer is to use these features for classifying the input image into various classes on the basis of the training dataset. ${ }^{75}$

algorithms before being incorporated into the machine algorithm. By contrast, deep learning mainly relies on large datasets to generate data representations rather than feature-specific algorithms. This approach allows deep learning algorithms to programme autonomously by learning from a large set of examples that demonstrate the desired behaviour. The need to specify rules explicitly is thereby removed, allowing unsupervised learning as the relevant features are automatically learned.

One widely used deep learning model is convolutional neural networks (CNNs). CNNs can take in an input image and assign importance to various features to achieve the desired outcome and behaviour (figure 3). Several studies have shown increased sensitivity and specificity when CNNs were used for the detection of diabetic retinopathy. ${ }^{78}$ Using a CNN in microaneurysm detection shows a sensitivity value of 0.8 for every mean of more than six false positives per image. ${ }^{79}$ The automated analysis of retinal colour images for diabetic retinopathy detection has been studied extensively, ${ }^{80-82}$ with systems in clinical use in both Europe and the USA. Apart from fundus images, CNN models have been used in spectral domain optical coherence tomography segmentation models to identify hyperreflective foci that surpass the accuracy of traditional methods. ${ }^{83}$

Initially, automated retinal image analysis algorithm development was confined to small (usually start-up) software firms. The potential market size and growth potential, however, have led to a myriad of entrantsincluding industry-leading players such as Google and IBM-investing considerable resources into these developments.

Currently, systems for the automated and computerassisted detection, classification, and diagnosis of diabetic retinopathy vary greatly in design, validation, degree of autonomy, and clinical use. Several automated and computer-assisted approaches can be used to detect diabetic retinopathy and monitor patients who are at risk for diabetic retinopathy progression and vision loss.

Nevertheless, while artificial intelligence technologies are in the process of resolving clinical and costeffectiveness challenges as well as image acquisition and quality concerns, the extent of acceptance by both patients and health-care professionals, as well as unresolved medicolegal questions, limit implementation in most countries at present. ${ }^{84}$

\section{Predictive risk models for diabetic retinopathy progression}

Models for predicting the risk of developing diabetic retinopathy and its progression are based on the creation of a learning system that enables aggregation and analysis of the great wealth of diverse patient conditions and treatment approaches taken by eye-care providers. The increased use of electronic medical records has resulted in the creation of large high-resolution health information databases that can be used in the development of deep learning or artificial intelligence models. This approach has resulted in the concept of a more personalised medicine with the goal of providing the right treatment to the right patient at the right time. With the use of predictive modelling, a wide array of factors (eg, clinical care, genomics, metabolomics, proteomics, imaging, etc) and a highly complex physician decision-making process will be integrated. Such work can potentially optimise care of complex chronic diseases such as diabetes and predict the risk of developing diabetic retinopathy in a personalised way.

Application of predictive modelling promises several innovative approaches that use a multidisciplinary approach to diabetes care, leveraging the clinical expertise of eye-care providers to potentially facilitate the use of the model in day-to-day clinical practice. Unique and complex clinical scenarios that would not be easily examined or not financially available in randomised clinical trials can be addressed through this collaborative clinician-engineer process..$^{85,86}$

\section{Retinal imaging in low-resource settings-handheld mobile devices}

Western Australia and Surabaya, Indonesia, were the first regions to implement use of handheld retinal cameras to provide community-based clinical examinations for diabetic retinopathy detection in lowresource settings. Ian Constable and colleagues from the Lions Eye Institute in Australia reported one of the first projects to establish these remote diabetic retinopathy assessments in $2000 .^{87}$ A major limitation at the time was that the imaging resolution was far inferior to those currently available. However, the programme did show the potential for such systems to extend eye care to lowresource settings and difficult-to-reach locations. The retinal images not only served as the patient's individual clinical record, but also substantially supported the 
education of patients and staff and formed the basis of initial research into such methods. Although many of the aspirations of those original projects have yet to be fully realised on a wider scale, subsequent technological developments, including new portable imaging devices that are readily available and fairly cheap (eg, based on smartphone technologies), should advance this agenda. Such technologies are easy to use and have validated sensitivity and specificity. Therefore, their implementation seems to be both feasible and realistic. ${ }^{88-91}$

Several studies have shown that eye diseases related to diabetes can be prevented through early detection and screening, and retinal assessments help in the prevention of vision loss resulting from diabetic retinopathy. However, the increasing number of patients with diabetes-related ocular complications makes retinal assessment for diabetic retinopathy detection unfeasible, as this assessment involves in-person examination of the patient. ${ }^{83}$ Hence, diabetic retinopathy assessment through telemedicine is a practical approach to prevent vision loss caused by diabetic retinopathy. To make the utilisation of telemedicine for diabetic retinopathy screening efficient, retinal specialists and image graders assess retinal images at reading centres, then referrals are made depending on the results of the graded images. ${ }^{92}$

Globally, there are not enough skilled human readers of retinal images to meet current-let alone futuredemands. To meet this need, software manufacturers have developed or modified existing artificial intelligence systems to support the detection, differentiation, and ultimately the classification or triaging of retinal conditions, particularly diabetic retinopathy. Through pattern recognition and image classification used by artificial intelligence systems, assessment of retinal images for diabetic retinopathy screening can be made possible. ${ }^{75}$

As the rates of development of information technology and digital imaging increase, we are now finally in a position where image capture, transmission, and storage potentially support practical telemedicine and, ultimately, various forms of automated analysis. Telemedicine for diabetic retinopathy has historically been hampered by insufficiencies in all of these areas, and has been far from cost-effective, certainly in low-resource settings, while generating little valuable data.

A more effective use of appropriate digital retinal imaging coupled with telemedicine to transmit images (and return reports) is urgently required to ensure equitable access to eye assessments in low-resource settings. Similarly, the application of automated analysis and artificial intelligence systems will increasingly have a place in supporting developments in both low-income and higher-income settings.

\section{Screening for retinal neurodegeneration}

Diabetic retinopathy has historically been considered to be an exclusively microvascular condition. In the last
10-15 years, this concept has shifted to an understanding that it is a more complex complication that involves retinal neurodegeneration. ${ }^{93-95}$ Indeed, the American Diabetes Association has redefined diabetic retinopathy as a "highly tissue-specific neurovascular complication". ${ }^{34}$ At present, there is no evidence that retinal neurodegeneration is related to the development and progression of microvascular disease. This issue should be investigated in future long-term clinical trials with highly sensitive new technologies combined with improved stratification of patients. Nevertheless, since the neuron loss that occurs in the diabetic retina is related to deficient sensory capacity and vision-related quality of life, periodic assessments of neurodegeneration or neurodysfunction in people with diabetes have been recommended. . $^{94,96}$

Several methods exist for measuring retinal neurodegeneration. Multifocal electroretinography (mfERG) and spectral domain optical coherence tomography are among the current methods used for this purpose. These non-invasive technologies allow the detection of functional (ie, delayed P1 implicit time and decreased amplitude) and morphological (ie, thinning of inner retinal layers and the nerve fibre layer) retinal abnormalities, respectively. Notably, neurodysfunction precedes the morphological changes in the diabetic retina. ${ }^{97,98}$ Therefore, tests to detect functional changes will identify early stages of retinal neurodegeneration, which could potentially be reversible. However, mfERG is a cumbersome and time-consuming procedure requiring specialised staff. Thus, it is not suitable for screening of retinal neurodegeneration and is mainly reserved for clinical trials. In the past 5-10 years, microperimetry has emerged as a simple test with even higher sensitivity than mfERG for detecting early functional changes of the retina, and can be used for screening.9 ${ }^{9}$ Furthermore, a full-field flicker electroretinogram from a handheld recording device has been used successfully for detecting early neurodysfunction in patients with diabetes who do not have clinically detectable retinopathy, and early neurodysfunction detected in this way has been linked to high $\mathrm{HbA}_{1 \mathrm{c}}$ levels. ${ }^{100}$

Finally, in experimental studies, GLP-1 receptor agonists $^{101,102}$ and DPP-4 inhibitors, ${ }^{103}$ provided both neuroprotection and prevention of vascular leakage. These findings open up a possible scenario in which the screening for retinal neurodysfunction could be considered essential for identifying a subset of patients in whom neuroprotective treatment might be beneficial.

\section{Retinopathy screening and other diabetes complications}

Apart from retinal neurovascular disease, the presence of diabetic retinopathy means that microcirculation has already been damaged by the diabetic milieu. Therefore, diabetic retinopathy can be considered a reliable marker of the deleterious effects of diabetes in an individual. Accordingly, diabetic retinopathy identifies a subset of 
Search strategy and selection criteria

We searched PubMed and Google Scholar for articles published in English from database inception up to Nov 10, 2019, using the search terms "diabetic retinopathy", "screening of diabetic retinopathy", "retinal imaging", "retinal neurodegeneration", "tele-ophthalmology", "artificial intelligence", "diabetic complications", "diabetic retinopathy and cardiovascular disease", and "diabetic retinopathy and dementia" (alone and in combination). We also searched the reference lists of original research articles, clinical guidelines, systematic reviews, and meta-analyses for further relevant material. Publications were selected on the basis of relevance.

the population with diabetes that is at a high risk of developing not only other microangiopathic complications (diabetic nephropathy and diabetic neuropathy, including diabetic foot), but also macrovascular outcomes. ${ }^{104,105}$ Notably, a population-based cohort study showed that the cumulative burden of microvascular disease increases the risk of future cardiovascular diseases among individuals with type 2 diabetes. ${ }^{106}$ However, although microalbuminuria and macroalbuminuria are frequently included among the risk factors in studies that assess risk of cardiovascular events, the presence and severity of diabetic retinopathy is often missing. This omission is a serious error because diabetic retinopathy is an independent risk factor for cardiovascular diseases and cardiovascular mortality. ${ }^{104}$

The increasing interest in the development of automated analysis software using deep neural learning based on artificial intelligence for analysis of retinal images makes it foreseeable that specific software will be developed to better define the cardiovascular risk in individuals with diabetes on the basis of the retinal structural and functional changes of the microvasculature. In fact, deep learning models trained on data obtained from retinal fundus images were shown to be able to predict cardiovascular risk factors and major cardiac events (area under the receiver operating characteristic curve $=70) .{ }^{107}$

Patients with type 2 diabetes have a demonstrably higher than average risk of developing neurodegenerative conditions, particularly cognitive dysfunction such as Alzheimer's disease. ${ }^{108}$ As the retina is embryonically a brain-derived tissue, the eye could provide an effective window into the brain, supporting easy, non-invasive investigation of neurodegenerative similarities between retina and brain. The measurement of the thickness of the neuroretina or retinal fibre layer by spectral domain optical coherence tomography, ${ }^{109}$ or the assessment of retinal sensitivity ${ }^{110}$ and gaze fixation ${ }^{111}$ by microperimetry, have been useful for identifying patients with type 2 diabetes who have mild cognitive impairment, which could be a prodromal stage of Alzheimer's disease. These findings open new perspectives on strategies for screening for diabetic retinopathy in individuals older than 60 years, as retinopathy screening might not be restricted to prevention of sight-threatening disease but might also be used to identify individuals at risk of severe cognitive decline. Importantly, cognitive impairment can affect treatment adherence and diabetes self-management, resulting in poor glycaemic control and an increased frequency of severe hypoglycaemia and hospital admissions. ${ }^{108}$ The early diagnosis of cognitive impairment enables a more personalised treatment approach that takes the cognitive capacity of patients into account, as recommended by the American Diabetes Association. ${ }^{112}$

\section{Conclusions}

Health-care affordability, quality, and accessibility for diabetic retinopathy screening are important factors in the prevention of blindness in populations at risk. The combination of automated retinal image analysis and telemedicine has the potential to substantially improve how diabetes eye care is delivered by providing automated real-time assessment in a more personalised way. Additionally, the introduction of new technologies for diabetic retinopathy screening will improve its costeffectiveness. Finally, the possibility of using retinal examination to help to identify patients at risk of cardiovascular disease and cognitive impairment could change the concept of diabetic retinopathy screening, with benefits beyond the prevention of sight-threatening disease.

\section{Contributors}

All authors are fully responsible for all content, were involved at all stages of writing and development of the Review (including the searches of the scientific literature), and approved the final submitted version.

\section{Declaration of interests}

We declare no competing interests.

\section{References}

1 Cheung N, Mitchell P, Wong TY. Diabetic retinopathy. Lancet 2010; 376: $124-36$

2 Yau JWY, Rogers SL, Kawasaki R, et al. Global prevalence and major risk factors of diabetic retinopathy. Diabetes Care 2012; 35: 556-64.

3 Wong TY, Mwamburi M, Klein R, et al. Rates of progression in diabetic retinopathy during different time periods: a systematic review and meta-analysis. Diabetes Care 2009; 32: 2307-13.

4 Flaxman SR, Bourne RRA, Resnikoff S, et al. Global causes of blindness and distance vision impairment 1990-2020: a systematic review and meta-analysis. Lancet Glob Health 2017; 5: e1221-34.

5 Li JQ, Welchowski T, Schmid M, et al. Prevalence, incidence and future projection of diabetic eye disease in Europe: a systematic review and meta-analysis. Eur J Epidemiol 2019; published online Sept 12. DOI:10.1007/s10654-019-00560-z.

6 Sabanayagam C, Banu R, Chee ML, et al. Incidence and progression of diabetic retinopathy: a systematic review. Lancet Diabetes Endocrinol 2019; 7: 140-49.

7 Lachin JM, Genuth S, Nathan DM, Zinman B, Rutledge BN, for the DCCT/EDIC Research Group. Effect of glycemic exposure on the risk of microvascular complications in the diabetes control and complications trial-revisited. Diabetes 2008; 57: 995-1001.

8 UK Prospective Diabetes Study (UKPDS) Group. Intensive blood-glucose control with sulphonylureas or insulin compared with conventional treatment and risk of complications in patients with type 2 diabetes (UKPDS 33). Lancet 1998; 352: 837-53.

9 Chew EY, Davis MD, Danis RP, et al. The effects of medical management on the progression of diabetic retinopathy in persons with type 2 diabetes: the Action to Control Cardiovascular Risk in Diabetes (ACCORD) Eye Study. Ophthalmology 2014; 121: 2443-51. 
10 Action to Control Cardiovascular Risk in Diabetes Follow-On (ACCORDION) Eye Study Group and the Action to Control Cardiovascular Risk in Diabetes Follow-On (ACCORDION) Study Group. Persistent effects of intensive glycemic control on retinopathy in type 2 diabetes in the Action to Control Cardiovascular Risk in Diabetes (ACCORD) follow-on study. Diabetes Care 2016; 39: 1089-100.

$11 \mathrm{Lu} \mathrm{J}, \mathrm{Ma} X$, Zhou J, et al. Association of time in range, as assessed by continuous glucose monitoring, with diabetic retinopathy in type 2 diabetes. Diabetes Care 2018; 41: 2370-76.

12 Zhao Q, Zhou F, Zhang Y, Zhou X, Ying C. Fasting plasma glucose variability levels and risk of adverse outcomes among patients with type 2 diabetes: a systematic review and meta-analysis. Diabetes Res Clin Pract 2019; 148: 23-31.

13 Arar NH, Freedman BI, Adler SG, et al. Heritability of the severity of diabetic retinopathy: the FIND-Eye study. Invest Ophthalmol Vis Sci 2008; 49: 3839-45.

14 Hietala K, Forsblom C, Summanen P, Groop P-H, on behalf of the FinnDiane Study Group. Heritability of proliferative diabetic retinopathy. Diabetes 2008; 57: 2176-80.

15 Hallman DM, Huber JC Jr, Gonzalez VH, Klein BE, Klein R, Hanis CL. Familial aggregation of severity of diabetic retinopathy in Mexican Americans from Starr County, Texas. Diabetes Care 2005; 28: 1163-68.

16 Monti MC, Lonsdale JT, Montomoli C, Montross R, Schlag E, Greenberg DA. Familial risk factors for microvascular complications and differential male-female risk in a large cohort of American families with type 1 diabetes. J Clin Endocrinol Metab 2007; 92: $4650-55$.

17 Scanlon PH. Update on screening for sight-threatening diabetic retinopathy. Ophthalmic Res 2019; 62: 218-24.

18 Nguyen HV, Tan GS, Tapp RJ, et al. Cost-effectiveness of a national telemedicine diabetic retinopathy screening program in Singapore. Ophthalmology 2016; 123: 2571-80.

19 Rachapelle S, Legood R, Alavi Y, et al. The cost-utility of telemedicine to screen for diabetic retinopathy in India. Ophthalmology 2013; 120: $566-73$.

20 Scanlon PH. Screening intervals for diabetic retinopathy and implications for care. Curr Diab Rep 2017; 17: 96.

21 Jones CD, Greenwood RH, Misra A, Bachmann MO. Incidence and progression of diabetic retinopathy during 17 years of a populationbased screening program in England. Diabetes Care 2012; 35: 592-96.

22 Agardh E, Tababat-Khani P. Adopting 3-year screening intervals for sight-threatening retinal vascular lesions in type 2 diabetic subjects without retinopathy. Diabetes Care 2011; 34: 1318-19.

23 Looker HC, Nyangoma SO, Cromie DT, et al. Predicted impact of extending the screening interval for diabetic retinopathy: the Scottish Diabetic Retinopathy Screening programme. Diabetologia 2013; 56: 1716-25.

24 Vujosevic S, Pucci P, Casciano M, et al. A decade-long telemedicine screening program for diabetic retinopathy in the north-east of Italy. J Diabetes Complications 2017; 31: 1348-53.

25 Groeneveld Y, Tavenier D, Blom JW, Polak BCP. Incidence of sight-threatening diabetic retinopathy in people with type 2 diabetes mellitus and numbers needed to screen: a systematic review. Diabet Med 2019; 36: 1199-208.

26 Lund SH, Aspelund T, Kirby P, et al. Individualised risk assessmen for diabetic retinopathy and optimisation of screening intervals: a scientific approach to reducing healthcare costs. $\mathrm{Br} J$ Ophthalmol 2016; 100: 683-87.

27 Stratton IM, Aldington SJ, Taylor DJ, Adler AI, Scanlon PH. A simple risk stratification for time to development of sight-threatening diabetic retinopathy. Diabetes Care 2013; 36: $580-85$.

28 Tufail A, Kapetanakis VV, Salas-Vega S, et al. An observational study to assess if automated diabetic retinopathy image assessment software can replace one or more steps of manual imaging grading and to determine their cost-effectiveness. Health Technol Assess 2016; 20: $1-72$.

29 van der Heijden AA, Abramoff MD, Verbraak F, van Hecke MV, Liem A, Nijpels G. Validation of automated screening for referable diabetic retinopathy with the IDx-DR device in the Hoorn Diabetes Care System. Acta Ophthalmol 2018; 96: 63-68.
30 Silva PS, Cavallerano JD, Haddad NM, et al. Peripheral lesions identified on ultrawide field imaging predict increased risk of diabetic retinopathy progression over 4 years. Ophthalmology 2015; 122: 949-56.

31 Fenner BJ, Wong RLM, Lam WC, Tan GSW, Cheung GCM. Advances in retinal imaging and applications in diabetic retinopathy screening: a review. Ophthalmol Ther 2018; 7: 333-46.

32 Leal J, Luengo-Fernandez R, Stratton IM, Dale A, Ivanova K, Scanlon PH. Cost-effectiveness of digital surveillance clinics with optical coherence tomography versus hospital eye service follow-up for patients with screen-positive maculopathy. Eye (Lond) 2019; 33: $640-47$

33 Wong TY, Sun J, Kawasaki R, et al. Guidelines on diabetic eye care: the International Council of Ophthalmology recommendations for screening, follow-up, referral, and treatment based on resource settings. Ophthalmology 2018; 125: 1608-22.

34 Solomon SD, Chew E, Duh EJ, et al. Diabetic retinopathy: a position statement by the American Diabetes Association. Diabetes Care 2017; 40: 412-18.

35 Pugh JA, Jacobson JM, Van Heuven WA, et al. Screening for diabetic retinopathy: the wide-angle retinal camera. Diabetes Care 1993; 16: 889-95

36 Harding SP, Broadbent DM, Neoh C, White MC, Vora J. Sensitivity and specificity of photography and direct ophthalmoscopy in screening for sight threatening eye disease: the Liverpool Diabetic Eye Study. BMJ 1995; 311: 1131-35.

37 Lee JC, Nguyen L, Hynan LS, Blomquist PH. Comparison of 1-field, 2-fields, and 3-fields fundus photography for detection and grading of diabetic retinopathy. J Diabetes Complications 2019; 33: 107441

38 Boucher MC, Gresset JA, Angioi K, Olivier S. Effectiveness and safety of screening for diabetic retinopathy with two nonmydriatic digital images compared with the seven standard stereoscopic photographic fields. Can J Ophthalmol 2003; 38: 557-68.

39 Bursell SE, Cavallerano JD, Cavallerano AA, et al. Stereo nonmydriatic digital-video color retinal imaging compared with Early Treatment Diabetic Retinopathy Study seven standard field 35-mm stereo color photos for determining level of diabetic retinopathy. Ophthalmology 2001; 108: 572-85.

40 Vujosevic S, Benetti E, Massignan F, et al. Screening for diabetic retinopathy: 1 and 3 nonmydriatic 45-degree digital fundus photographs vs 7 standard early treatment diabetic retinopathy study fields. Am J Ophthalmol 2009; 148: 111-18.

41 Scanlon PH, Malhotra R, Greenwood RH, et al. Comparison of two reference standards in validating two field mydriatic digital photography as a method of screening for diabetic retinopathy. Br J Ophthalmol 2003; 87: 1258-63.

42 Williams GA, Scott IU, Haller JA, Maguire AM, Marcus D, McDonald HR. Single-field fundus photography for diabetic retinopathy screening: a report by the American Academy of Ophthalmology. Ophthalmology 2004; 111: 1055-62.

43 Aptel F, Denis P, Rouberol F, Thivolet C. Screening of diabetic retinopathy: effect of field number and mydriasis on sensitivity and specificity of digital fundus photography. Diabetes Metab 2008 34: 290-93.

44 Scanlon PH. The English national screening programme for sight-threatening diabetic retinopathy. J Med Screen 2008; 15: 1-4.

45 Silva PS, Cavallerano JD, Sun JK, Soliman AZ, Aiello LM, Aiello LP. Peripheral lesions identified by mydriatic ultrawide field imaging: distribution and potential impact on diabetic retinopathy severity. Ophthalmology 2013; 120: 2587-95.

46 Wilson JMG, Jungner G. Principles and practice of screening for disease. Geneva: World Health Organization, 1968. https://apps. who.int/iris/bitstream/handle/10665/37650/WHO_PHP_34.pdf (accessed Sept 12, 2019).

47 Scanlon PH. The English National Screening Programme for diabetic retinopathy 2003-2016. Acta Diabetol 2017; 54: 515-25.

48 Graham-Rowe E, Lorencatto F, Lawrenson JG, et al. Barriers to and enablers of diabetic retinopathy screening attendance: a systematic review of published and grey literature. Diabet Med 2018; 35: 1308-19.

49 Lawrenson JG, Graham-Rowe E, Lorencatto F, et al. What works to increase attendance for diabetic retinopathy screening? An evidence synthesis and economic analysis. Health Technol Assess 2018; 22: $1-160$. 
50 Leese GP, Boyle P, Feng Z, Emslie-Smith A, Ellis JD. Screening uptake in a well-established diabetic retinopathy screening program: the role of geographical access and deprivation. Diabetes Care 2008; 31: 2131-35.

51 Scanlon PH, Stratton IM, Leese GP, et al. Screening attendance, age group and diabetic retinopathy level at first screen. Diabet Med 2016; 33: 904-11.

52 Forster AS, Forbes A, Dodhia H, et al. Non-attendance at diabetic eye screening and risk of sight-threatening diabetic retinopathy: a population-based cohort study. Diabetologia 2013; 56: 2187-93.

53 Cavan D, Makaroff LE, da Rocha Fernandes J, et al. Global perspectives on the provision of diabetic retinopathy screening and treatment: survey of health care professionals in 41 countries. Diabetes Res Clin Pract 2018; 143: 170-78.

54 Danielsen R, Jónasson F, Helgason T. Prevalence of retinopathy and proteinuria in type 1 diabetics in Iceland. Acta Med Scand 1982; 212: $277-80$.

55 Kristinsson JK, Hauksdóttir H, Stefánsson E, Jónasson F, Gíslason I. Active prevention in diabetic eye disease. A 4-year follow-up. Acta Ophthalmol Scand 1997; 75: 249-54.

56 Gillow JT, Gray JA. The National Screening Committee review of diabetic retinopathy screening. Eye (Lond) 2001; 15: 1-2.

57 Diabetic Retina Screen. Diabetic RetinaScreen-The National Diabetic Retinal Screening Programme.

http://www.diabeticretinascreen.ie/ (accessed July 26, 2019).

58 Kohner EM, Porta M. Protocols for screening and treatment of diabetic retinopathy in Europe. Eur J Ophthalmol 1991; 1: 45-54.

59 Anon. The Liverpool Declaration on screening for diabetic retinopathy in Europe. http://www.drscreening2005.org.uk/ declaration_2005.html (accessed Sept 10, 2019).

60 Anon. Screening for diabetic retinopathy in Europe-strategies for overcoming hurdles to progress. http://www.drscreening2005.org. uk/gdansk_2011.html (accessed Feb 3, 2019).

61 Broadbent DM, Sampson CJ, Wang A, et al. Individualised screening for diabetic retinopathy: the ISDR study-rationale, design and methodology for a randomised controlled trial comparing annual and individualised risk-based variable-interval screening. BMJ Open 2019; 9: e025788.

62 Scanlon PH, Aldington SJ, Leal J, et al. Development of a cost-effectiveness model for optimisation of the screening interval in diabetic retinopathy screening. Health Technol Assess 2015; 19: $1-116$.

63 Leese GP, Stratton IM, Land M, et al. Progression of diabetes retinal status within community screening programs and potential implications for screening intervals. Diabetes Care 2015; 38: 488-94.

64 Taylor-Phillips S, Mistry H, Leslie R, et al. Extending the diabetic retinopathy screening interval beyond 1 year: systematic review. Br J Ophthalmol 2016; 100: 105-14.

65 Public Health England. Diabetic eye screening key performance indicators for 2018 to 2019: definitions. 2018. https://www.gov.uk/ government/publications/nhs-population-screening-reportingdata-definitions/diabetic-eye-screening-key-performance-indicatorsfor-2018-to-2019-definitions (accessed Feb 17, 2020).

66 Scanlon PH, Aldington SJ, Stratton IM. Delay in diabetic retinopathy screening increases the rate of detection of referable diabetic retinopathy. Diabet Med 2014; 31: 439-42.

67 Pasquel FJ, Hendrick AM, Ryan M, Cason E, Ali MK, Narayan KM. Cost-effectiveness of different diabetic retinopathy screening modalities. J Diabetes Sci Technol 2015; 10: 301-07.

68 Orton E, Forbes-Haley A, Tunbridge L, Cohen S. Equity of uptake of a diabetic retinopathy screening programme in a geographically and socio-economically diverse population. Public Health 2013; 127: 814-21.

69 Mamtora S, Sandinha T, Carey PE, Steel DHW. Optimizing medical management in patients with sight-threatening diabetic retinopathy. Ophthalmol Ther 2017; 6: 105-14.

70 Wong TY, Sabanayagam C. Strategies to tackle the global burden of diabetic retinopathy: from epidemiology to artificial intelligence. Ophthalmologica 2019; published online Aug 13. DOI:10.1159/000502387.

71 International Agency for the Prevention of Blindness. A global compendium on good practice: integrated care for diabetes and eye health. 2018. https://www.iapb.org/resources/ a-global-compendium on-good-practice-integrated-care-for-diabetes-and-eye-health/ (accessed Nov 10, 2019).
72 Piyasena MMPN, Murthy GVS, Yip JLY, et al. Systematic review on barriers and enablers for access to diabetic retinopathy screening services in different income settings. PLoS One 2019; 14: e0198979.

73 Silva PS, Cavallerano JD, Sun JK, Noble J, Aiello LM, Aiello LP. Nonmydriatic ultrawide field retinal imaging compared with dilated standard 7-field 35-mm photography and retinal specialist examination for evaluation of diabetic retinopathy. Am J Ophthalmol 2012; 154: 549-559.e2.

74 Silva PS, Horton MB, Clary D, et al. Identification of diabetic retinopathy and ungradable image rate with ultrawide field imaging in a national teleophthalmology program. Ophthalmology 2016; 123: 1360-67.

75 Cheung CY, Tang F, Ting DSW, Tan GSW, Wong TY. Artificia intelligence in diabetic eye disease screening. Asia Pac J Ophthalmol (Phila) 2019; 8: 158-64.

76 Padhy SK, Takkar B, Chawla R, Kumar A. Artificial intelligence in diabetic retinopathy: a natural step to the future. Indian J Ophthalmol 2019; 67: 1004-09.

77 Wong TY, Bressler NM. Artificial intelligence with deep learning technology looks into diabetic retinopathy screening. JAMA 2016 316: $2366-67$.

78 Lundervold AS, Lundervold A. An overview of deep learning in medical imaging focusing on MRI. Z Med Phys 2019; 29: 102-27.

79 Eftekhari N, Pourreza HR, Masoudi M, Ghiasi-Shirazi K, Saeedi E. Microaneurysm detection in fundus images using a two-step convolutional neural network. Biomed Eng Online 2019; 18 : 67.

80 Abràmoff MD, Folk JC, Han DP, et al. Automated analysis of retinal images for detection of referable diabetic retinopathy. JAMA Ophthalmol 2013; 131: 351-57.

81 Ege BM, Hejlesen OK, Larsen OV, et al. Screening for diabetic retinopathy using computer based image analysis and statistical classification. Comput Methods Programs Biomed 2000; 62: 165-75.

82 Trucco E, Ruggeri A, Karnowski T, et al. Validating retinal fundus image analysis algorithms: issues and a proposal. Invest Ophthalmol Vis Sci 2013; 54: 3546-59.

83 Yu C, Xie S, Niu S, et al. Hyper-reflective foci segmentation in SD-OCT retinal images with diabetic retinopathy using deep convolutional neural networks. Med Phys 2019; 46: 4502-19.

84 Ting DSW, Lee AY, Wong TY. An ophthalmologist's guide to deciphering studies in artificial intelligence. Ophthalmology 2019; 126: $1475-79$.

85 Cosgriff CV, Celi LA, Ko S, et al. Developing well-calibrated illness severity scores for decision support in the critically ill. NPJ Digit Med 2019; 2: 76.

86 Celi LA, Hinske LC, Alterovitz G, Szolovits P. An artificial intelligence tool to predict fluid requirement in the intensive care unit: a proof-of-concept study. Crit Care 2008; 12: R151.

87 Constable IJ, Yogesan K, Eikelboom R, Barry C, Cuypers M. Fred Hollows lecture: digital screening for eye disease. Clin Exp Ophthalmol 2000; 28: 129-32.

88 Bilong Y, Katte JC, Koki G, et al. Validation of smartphone-based retinal photography for diabetic retinopathy screening. Ophthalmic Surg Lasers Imaging Retina 2019; 50: S18-22.

89 Toy BC, Myung DJ, He L, et al. Smartphone-based dilated fundus photography and near visual acuity testing as inexpensive screening tools to detect referral warranted diabetic eye disease. Retina 2016; 36: $1000-08$.

90 Russo A, Morescalchi F, Costagliola C, Delcassi L, Semeraro F. Comparison of smartphone ophthalmoscopy with slit-lamp biomicroscopy for grading diabetic retinopathy. Am J Ophthalmol 2015; 159: 360-64.e1.

91 Rajalakshmi R, Arulmalar S, Usha M, et al. Validation of smartphone based retinal photography for diabetic retinopathy screening. PLoS One 2015; 10: e0138285.

92 Salongcay RP, Silva PS. The role of teleophthalmology in the management of diabetic retinopathy. Asia Pac J Ophthalmol (Phila) 2018; 7: 17-21.

93 Simó R, Hernández C. Neurodegeneration in the diabetic eye: new insights and therapeutic perspectives. Trends Endocrinol Metab 2014; 25: $23-33$

94 Simó R, Stitt AW, Gardner TW. Neurodegeneration in diabetic retinopathy: does it really matter? Diabetologia 2018; 61: 1902-12. 
95 Sohn EH, van Dijk HW, Jiao C, et al. Retinal neurodegeneration may precede microvascular changes characteristic of diabetic retinopathy in diabetes mellitus. Proc Natl Acad Sci USA 2016; 113: E2655-64.

96 Abramoff MD, Fort PE, Han IC, Jayasundera KT, Sohn EH, Gardner TW. Approach for a clinically useful comprehensive classification of vascular and neural aspects of diabetic retinal disease. Invest Ophthalmol Vis Sci 2018; 59: 519-27.

97 Santos AR, Ribeiro L, Bandello F, et al. Functional and structural findings of neurodegeneration in early stages of diabetic retinopathy: cross-sectional analyses of baseline data of the EUROCONDOR project. Diabetes 2017; 66: 2503-10.

98 Simó R, Hernández C, Porta M, et al. Effects of topically administered neuroprotective drugs in early stages of diabetic retinopathy: results of the EUROCONDOR clinical trial. Diabetes 2019; 68: 457-63.

99 Wu Z, Ayton LN, Guymer RH, Luu CD. Comparison between multifocal electroretinography and microperimetry in age-related macular degeneration. Invest Ophthalmol Vis Sci 2014; 55: 6431-39.

100 Zeng Y, Cao D, Yu H, et al. Early retinal neurovascular impairment in patients with diabetes without clinically detectable retinopathy. Br J Ophthalmol 2019; 103: 1747-52.

101 Hernández C, Bogdanov P, Corraliza L, et al. Topical administration of GLP-1 receptor agonists prevents retinal neurodegeneration in experimental diabetes. Diabetes 2016; 65: 172-87.

102 Sampedro J, Bogdanov P, Ramos H, et al. New insights into the mechanisms of action of topical administration of GLP-1 in an experimental model of diabetic retinopathy. J Clin Med 2019; 8: E339.

103 Hernández C, Bogdanov P, Solà-Adell C, et al. Topical administration of DPP-IV inhibitors prevents retinal neurodegeneration in experimental diabetes. Diabetologia 2017; 60: 2285-98.

104 Pearce I, Simó R, Lövestam-Adrian M, Wong DT, Evans M. Association between diabetic eye disease and other complications of diabetes: implications for care. A systematic review. Diabetes Obes Metab 2019; 21: 467-78.
105 Rosenson RS, Fioretto P, Dodson PM. Does microvascular disease predict macrovascular events in type 2 diabetes? Atherosclerosis 2011; 218: $13-18$.

106 Brownrigg JRW, Hughes CO, Burleigh D, et al. Microvascular disease and risk of cardiovascular events among individuals with type 2 diabetes: a population-level cohort study. Lancet Diabetes Endocrinol 2016; 4: 588-97.

107 Poplin R, Varadarajan AV, Blumer K, et al. Prediction of cardiovascular risk factors from retinal fundus photographs via deep learning. Nat Biomed Eng 2018; 2: 158-64.

108 Simó R, Ciudin A, Simó-Servat O, Hernández C. Cognitive impairment and dementia: a new emerging complication of type 2 diabetes-the diabetologist's perspective. Acta Diabetol 2017; 54: 417-24.

109 Cheung CY, Ikram MK, Chen C, Wong TY. Imaging retina to study dementia and stroke. Prog Retin Eye Res 2017; 57: 89-107.

110 Ciudin A, Simó-Servat O, Hernández C, et al. Retinal microperimetry: a new tool for identifying patients with type 2 diabetes at risk for developing Alzheimer disease. Diabetes 2017; 66: 3098-104.

111 Simó-Servat O, Ciudin A, Ortiz-Zúñiga ÁM, Hernández C, Simó R. Usefulness of eye fixation assessment for identifying type 2 diabetic subjects at risk of dementia. J Clin Med 2019; 8: 59.

112 American Diabetes Association. Summary of revisions for the 2013 clinical practice recommendations. Diabetes Care 2013; 36 (suppl 1): S3.

(C) 2020 Elsevier Ltd. All rights reserved. 